\title{
First-Principles Calculation of the Air-Water Second Virial Coefficient
}

\author{
A. H. Harvey ${ }^{1,2}$ and P. H. Huang ${ }^{3}$
}

\begin{abstract}
Recent theoretical work has produced quantitatively accurate potential-energy surfaces for water with common gases. These pair potentials have been used to calculate second interaction virial coefficients with an accuracy superior to that obtained by most experiments. In this work, results for water-nitrogen, water-oxygen, and water-argon are combined to calculate an effective second virial coefficient for water with air. The results are in agreement with the existing experimental data, but they cover a wide range of temperatures while the experimental data extend only from 253 to $348 \mathrm{~K}$. These results will be useful for humidity standards and other applications requiring thermodynamic properties of moist air.
\end{abstract}

KEY WORDS: air; enthalpy coefficient; humidity; second virial coefficient; water.

\section{INTRODUCTION}

Many applications in science and industry require a reliable description of water content in gases. For quantitative accuracy, especially at higher pressures, it is necessary to consider the deviation of the equilibrium moisture content from that given by an ideal calculation based only on the vapor pressure of water. In most cases, the dominant correction is the deviation of the vapor-phase thermodynamic behavior from that of an ideal gas. This nonideality can typically be approximated accurately at the level of

\footnotetext{
${ }^{1}$ Physical and Chemical Properties Division, National Institute of Standards and Technology, 325 Broadway, Boulder, Colorado 80305-3328, U.S.A.

${ }^{2}$ To whom correspondence should be addressed. E-Mail: aharvey@boulder.nist.gov

${ }^{3}$ Process Measurements Division, National Institute of Standards and Technology, 100 Bureau Drive, Gaithersburg, Maryland 20899-8363, U.S.A.
} 
the second virial coefficient, which is the first-order correction to the idealgas law. The key parameter is the cross-second virial coefficient, representing the interaction between water and the carrier gas.

In this work, we consider the important case where the carrier gas is air. The vapor-phase thermodynamics of air-water mixtures is important for applications such as humidity standards, hot-wire anemometry, industrial drying, and humid-air turbines for power generation. Recent studies $[1,2]$ have pointed to the lack of data for the air-water cross-second virial coefficient $B_{\mathrm{aw}}$ at high temperatures as an obstacle to accurate design calculations in industry.

The primary experimental data for $B_{\text {aw }}$ were produced by Hyland and Wexler [3,4] at temperatures from -20 to $70^{\circ} \mathrm{C}$, and by Wylie and Fisher [5] from 20 to $75^{\circ} \mathrm{C}$. These two sources agree within their mutual uncertainties at the temperatures where they overlap. Some older, less precise data sources are discussed by Hyland and Wexler [3]; these are also limited to a narrow temperature range.

Hyland and Wexler [6] correlated $B_{\text {aw }}$ as a function of temperature based on their data $[3,4]$ and on a preliminary report of the data of Wylie and Fisher [5]. This correlation is widely used in the humidity field. Its temperature dependence was chosen to be that of a Lennard-Jones fluid, which is not a realistic model for pair interactions involving water. Therefore, while the Hyland-Wexler correlation [6] is often extrapolated to high and low temperatures simply because no other approach is available [2], the reliability of such extrapolation is doubtful.

Alternatively, if the potential-energy surface between a pair of molecules is known, second virial coefficients may be calculated from statistical mechanics [7]. This calculation may be performed at any temperature, making it an attractive option for conditions where experimental data are lacking. Furthermore, computational quantum chemistry has reached the stage where it is possible to derive highly accurate potentials for pairs of relatively small molecules, such as water and the components of air. In this paper, we take this approach to compute $B_{\mathrm{aw}}(T)$ from first principles, comparing the results with experimental data and with the commonly used correlation of Hyland and Wexler [6].

\section{WATER WITH INDIVIDUAL GASES}

We use the cross-second virial coefficients calculated as functions of temperature by Hodges etal. [8] for $\mathrm{H}_{2} \mathrm{O}-\mathrm{Ar}$, by Tulegenov et al. [9] for $\mathrm{H}_{2} \mathrm{O}-\mathrm{N}_{2}$, and by Wheatley and Harvey [10] for $\mathrm{H}_{2} \mathrm{O}-\mathrm{O}_{2}$. All of these were accurately represented in the original papers by functions of the form, 
Table I. Parameters for Eq. (1) for Calculating $B(T)$ for Individual Water-Gas Systems (the $c_{i}$ have units of $\mathrm{cm}^{3} \cdot \mathrm{mol}^{-1}$ and the $d_{i}$ are dimensionless)

\begin{tabular}{lccc}
\hline Parameter & $\mathrm{H}_{2} \mathrm{O}-\mathrm{Ar}[8]$ & $\mathrm{H}_{2} \mathrm{O}-\mathrm{N}_{2}[9]$ & $\mathrm{H}_{2} \mathrm{O}-\mathrm{O}_{2}[10]$ \\
\hline$c_{1}$ & 96.1591 & 67.595 & 124.605 \\
$c_{2}$ & -211.074 & -249.83 & -214.421 \\
$c_{3}$ & -96.4425 & -204.38 & -102.818 \\
$c_{4}$ & -12.6006 & - & -22.36 \\
$d_{1}$ & -0.31 & -0.24 & -0.33 \\
$d_{2}$ & -0.82 & -1.06 & -0.73 \\
$d_{3}$ & -2.24 & -3.22 & -2.03 \\
$d_{4}$ & -4.60 & - & -4.07 \\
\hline
\end{tabular}

$$
B(T)=\sum_{i=1}^{4} c_{i}\left(T^{*}\right)^{d_{i}}
$$

where $B$ is the second virial coefficient in $\mathrm{cm}^{3} \cdot \mathrm{mol}^{-1}, T^{*}=T /(100 \mathrm{~K})$, and $T$ is the temperature in $\mathrm{K}$. Table I lists the parameters $c_{i}$ and $d_{i}$ for each system. In all three cases, Eq. (1) covers a temperature range from $100 \mathrm{~K}$ up to at least $2000 \mathrm{~K}$.

For each of these systems, the developers of the potential-energy surface made estimates of its uncertainty, which they then propagated into calculations of $B(T)$ to produce uncertainties in that property. For this work, we have represented these uncertainties in $B$ (expanded uncertainty with a coverage factor $k=2$, denoted by $U(B)$ ) as functions of temperature:

$$
U(B)=\sum_{i=1}^{3} e_{i}\left(T^{*}\right)^{f_{i}},
$$

where $U(B)$ is in $\mathrm{cm}^{3} \cdot \mathrm{mol}^{-1}$ and $T^{*}=T /(100 \mathrm{~K})$. Table II lists the parameters $e_{i}$ and $f_{i}$ for each system.

\section{AIR-WATER MIXTURE}

We consider dry air to have the standard composition as given by Giacomo [11]. When minor components (those other than $\mathrm{N}_{2}, \mathrm{O}_{2}$, and Ar) are omitted and the compositions are renormalized, the resulting mole fractions are $x_{\mathrm{N}_{2}}=0.78135, x_{\mathrm{O}_{2}}=0.20948$, and $x_{\mathrm{Ar}}=0.00917$. 
Table II. Parameters for Eq. (2) for Calculating Expanded Uncertainties $U(B)$ for Individual Water-Gas Systems (the $e_{i}$ have units of $\mathrm{cm}^{3} \cdot \mathrm{mol}^{-1}$ and the $f_{i}$ are dimensionless)

\begin{tabular}{llll}
\hline Parameter & $\mathrm{H}_{2} \mathrm{O}-\mathrm{Ar}$ & $\mathrm{H}_{2} \mathrm{O}-\mathrm{N}_{2}$ & $\mathrm{H}_{2} \mathrm{O}-\mathrm{O}_{2}$ \\
\hline$e_{1}$ & 2.122 & 10.148 & 1.1073 \\
$e_{2}$ & 8.719 & 16.811 & 16.094 \\
$e_{3}$ & 4.983 & 25.819 & 12.33 \\
$f_{1}$ & -0.35 & -1.0 & -0.213 \\
$f_{2}$ & -1.5 & -2.15 & -1.3 \\
$f_{3}$ & -4.2 & -4.15 & -3.3 \\
\hline
\end{tabular}

The effective air-water second virial coefficient is simply a mole-fraction-weighted average of the contributions from the individual atmospheric gases:

$$
B_{\mathrm{aw}}(T)=\sum_{i=\mathrm{N}_{2}, \mathrm{O}_{2}, \mathrm{Ar}} x_{i} B_{i \mathrm{~W}}(T) .
$$

The expanded uncertainty $U\left(B_{\mathrm{aw}}\right)$ as a function of temperature is computed in a straightforward manner from the individual contributions:

$$
U\left(B_{\mathrm{aw}}\right)=\left(\sum_{i=\mathrm{N}_{2}, \mathrm{O}_{2}, \mathrm{Ar}} x_{i}\left[U\left(B_{i \mathrm{w}}\right)\right]^{2}\right)^{0.5} .
$$

Equation (4) assumes that uncertainties other than those in the individual gas-water $B(T)$ values are negligible; this assumption will be discussed in Section 5 .

While Eqs. (3) and (4) can be used directly to compute $B_{\text {aw }}$ and its uncertainty, it is more convenient to represent these results by single functions of the form of Eqs. (1) and (2). We therefore present the following functions:

$$
\begin{aligned}
B_{\mathrm{aw}}(T) /\left(1 \mathrm{~cm}^{3} \cdot \mathrm{mol}^{-1}\right)= & 66.5687\left(T^{*}\right)^{-0.237}-238.834\left(T^{*}\right)^{-1.048} \\
- & 176.755\left(T^{*}\right)^{-3.183} \\
U\left(B_{\mathrm{aw}}\right) /\left(1 \mathrm{~cm}^{3} \cdot \mathrm{mol}^{-1}\right)= & 3.9567\left(T^{*}\right)^{-0.8}+13.869\left(T^{*}\right)^{-1.65} \\
+ & 23.786\left(T^{*}\right)^{-3.92}
\end{aligned}
$$

where $T^{*}=T /(100 \mathrm{~K})$.

Equations (5) and (6) are recommended for the temperature range from 150 to $2000 \mathrm{~K}$, which covers conditions of practical interest where 


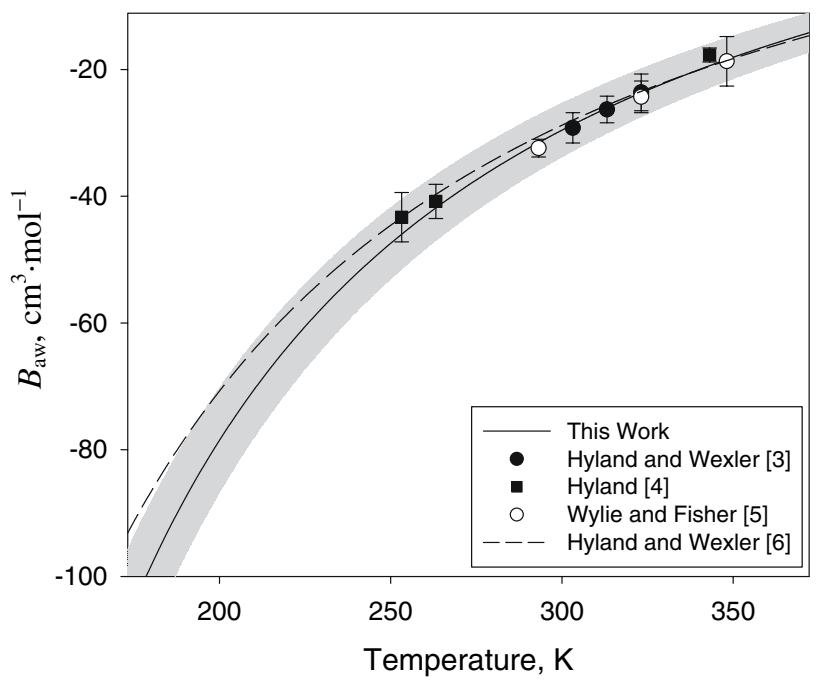

Fig. 1. Calculated values of $B_{\text {aw }}$ compared to experimental data and previous correlation. Shading represents expanded uncertainty of the present calculations.

$B_{\text {aw }}$ might be desired. Within this range, they fit the calculations of Eqs. (3) and (4) to better than $0.02 \mathrm{~cm}^{3} \cdot \mathrm{mol}^{-1}$. Equation (5) extrapolates reasonably down to $100 \mathrm{~K}$ and up to $3000 \mathrm{~K}$; further extrapolation is not recommended. Table III contains sample values of $B_{\text {aw }}$ and $U\left(B_{\mathrm{aw}}\right)$ computed from Eqs. (5) and (6).

In Fig. 1, we compare our calculations (where the shading represents the expanded $(K=2)$ uncertainty $\left.U\left(B_{\mathrm{aw}}\right)\right)$ with the available experimental data [3]-[5] and with the correlation of Hyland and Wexler [6]. Figure 1 covers only the temperature range (173.15-372.15 K) in which Hyland and Wexler recommended their correlation. In Fig. 2, we compare our results to the Hyland-Wexler correlation at higher temperatures. While this is beyond the range of validity recommended by Hyland and Wexler, it is not uncommon for their correlation to be extrapolated to such conditions, so it is useful to see how well it performs there.

\section{CALCULATION OF ENTHALPY COEFFICIENT}

The quantity $\phi_{\mathrm{aw}}=B_{\mathrm{aw}}-T \frac{\mathrm{d} B_{\mathrm{aw}}}{\mathrm{d} T}$, often called the enthalpy coefficient, appears in enthalpy and entropy calculations of air-water mixtures at the second virial coefficient level. The enthalpy coefficient $\phi_{\text {aw }}$ can be calculated directly from Eq. (5); values calculated in this manner are given in Table III. 


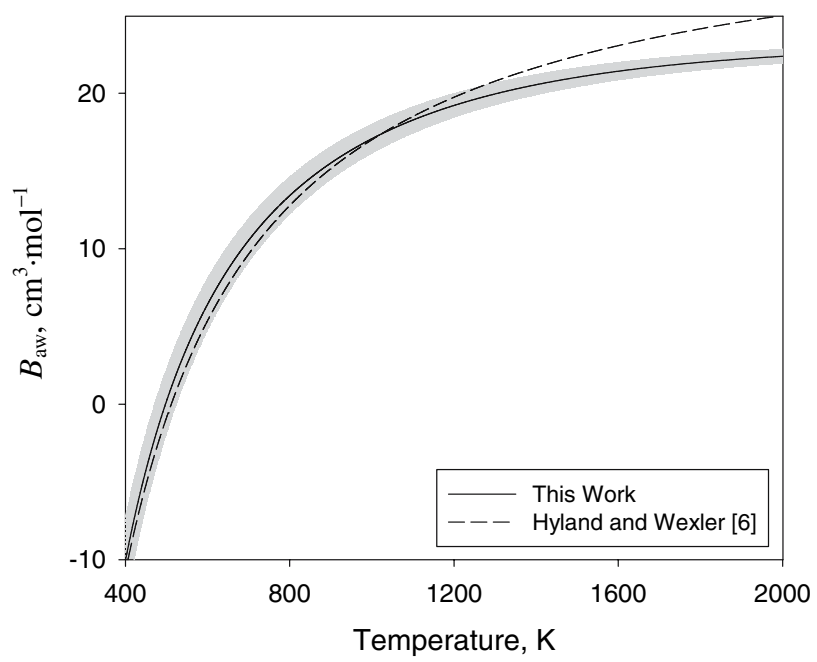

Fig. 2. Calculated values of $B_{\text {aw }}$ compared to extrapolation of Hyland-Wexler correlation [6] at high temperatures. Shading represents expanded uncertainty of the present calculations.

Table III. Second Virial Coefficients $B_{\mathrm{aw}}$, Expanded Uncertainties $U\left(B_{\mathrm{aw}}\right), \phi_{\mathrm{aw}}=B_{\mathrm{aw}}-$ $T \frac{\mathrm{d} B_{\mathrm{aw}}}{\mathrm{d} T}$, and Expanded Uncertainties $U\left(\phi_{\mathrm{aw}}\right)$ Calculated from Eqs. (5), (6), and (7) (units for all calculated quantities are $\mathrm{cm}^{3} \cdot \mathrm{mol}^{-1}$, and expanded uncertainties have coverage factor $k=$ 2)

\begin{tabular}{lrrrr}
\hline$T(\mathrm{~K})$ & \multicolumn{1}{c}{$B_{\text {aw }}$} & $U\left(B_{\text {aw }}\right)$ & \multicolumn{1}{c}{$\phi_{\text {aw }}$} & $U\left(\phi_{\text {aw }}\right)$ \\
\hline 150 & -144.31 & 14.82 & -448.41 & 47.73 \\
200 & -78.49 & 8.26 & -248.10 & 23.55 \\
250 & -47.41 & 5.61 & -160.98 & 14.77 \\
300 & -29.57 & 4.23 & -113.60 & 10.55 \\
350 & -18.07 & 3.38 & -84.12 & 8.13 \\
400 & -10.08 & 2.82 & -64.09 & 6.60 \\
450 & -4.24 & 2.41 & -49.63 & 5.53 \\
500 & 0.19 & 2.11 & -38.73 & 4.76 \\
600 & 6.42 & 1.69 & -23.42 & 3.71 \\
700 & 10.54 & 1.41 & -13.23 & 3.04 \\
800 & 13.41 & 1.21 & -6.02 & 2.57 \\
900 & 15.50 & 1.06 & -0.67 & 2.23 \\
1000 & 17.07 & 0.94 & 3.43 & 1.97 \\
1500 & 21.02 & 0.61 & 14.57 & 1.25 \\
2000 & 22.37 & 0.46 & 19.25 & 0.93 \\
\hline
\end{tabular}


Estimation of the expanded uncertainty $U\left(\phi_{\text {aw }}\right)$ is not trivial. In principle, the uncertainty in a quantity calculated from a pair potential can be estimated by calculating the quantity for all physically reasonable variations of the potential within boundaries defined by its uncertainty. For $B(T)$, this difficulty can be bypassed because the variation of the potential function that takes the maximum possible value at all points and the similar variation that takes minimum values at all points provide upper and lower bounds for all $B(T)$ that would be computed by any variation of the potential. The amount by which these maximum and minimum potentials change $B(T)$ therefore gives a conservative estimate for $U(B)$; the values of $U(B)$ for individual water-gas binaries [8-10], as correlated by Eq. (2) and used in this work, were obtained in this manner.

However, the maximum and minimum potentials do not necessarily provide bounds for $\phi(T)$. In order to estimate the range of variation in $\phi(T)$ for each water-gas binary, we evaluated $\phi(T)$ for four perturbations of each potential, chosen to represent extremes of possible variation. Two of these were the "maximum" and "minimum" potentials as described above. The other two were obtained by combining the "minimum" function at points where the potential takes positive values with the "maximum" where it takes negative values, and vice versa. From the shifts in $\phi(T)$ induced by these four variations, the largest positive and negative changes were noted at each temperature, and the mean of the magnitude of these two extremes was taken as our estimate of $U(\phi)$ for that temperature. The resulting expanded uncertainties $U(\phi)$ are larger than those obtained from simpler analysis in earlier work $[9,12]$, but only by a few percent.

The values of $U(\phi)$ for each water-gas binary were combined in a manner analogous to Eq. (4) to yield expanded uncertainties $U\left(\phi_{\mathrm{aw}}\right)$ for the air-water system. Because the $\mathrm{H}_{2} \mathrm{O}-\mathrm{Ar}$ potential [8] did not readily lend itself to our approach, we assumed that its $U(\phi)$ was identical to that for $\mathrm{H}_{2} \mathrm{O}-\mathrm{N}_{2}$. This is a conservative assumption, since $U(B)$ for $\mathrm{H}_{2} \mathrm{O}-\mathrm{Ar}$ is somewhat smaller than that for $\mathrm{H}_{2} \mathrm{O}-\mathrm{N}_{2}$; the small mole fraction of $\mathrm{Ar}$ in air makes $U\left(\phi_{\mathrm{aw}}\right)$ insensitive to this assumption in any case. $U\left(\phi_{\mathrm{aw}}\right)$ is accurately represented by the function,

$$
U\left(\phi_{\mathrm{aw}}\right) /\left(1 \mathrm{~cm}^{3} \cdot \mathrm{mol}^{-1}\right)=2.156\left(T^{*}\right)^{-0.52}+37.42\left(T^{*}\right)^{-1.46}+118.5\left(T^{*}\right)^{-3.81},
$$

where $T^{*}=T /(100 \mathrm{~K})$. Values of $U\left(\phi_{\mathrm{aw}}\right)$ calculated from Eq. (7) are given in Table III. 


\section{DISCUSSION}

Figure 1 shows that our calculated values of $B(T)$ are in excellent agreement with the experimental data. The agreement is as good as that of the Hyland-Wexler correlation [6], despite the fact that the correlation was fitted to those data, while our results are entirely a prediction. We agree, within our expanded uncertainty, with the correlation of Hyland and Wexler for almost its entire recommended range as shown in Fig. 1; only below about $190 \mathrm{~K}$ does their correlation stray outside our uncertainty band. It is worth noting that our temperature dependence below about $300 \mathrm{~K}$ is significantly different from that of Hyland and Wexler; this difference will affect calculations of enthalpy and entropy, since these involve $\mathrm{d} B / \mathrm{d} T$.

Figure 2 shows that, when extrapolated to high temperatures, the correlation of Hyland and Wexler continues to give reasonable results up to about $1200 \mathrm{~K}$. At still higher temperatures, the results diverge significantly. This is not unexpected, since the Hyland-Wexler correlation has little physical basis.

The expanded uncertainties $U\left(B_{\mathrm{aw}}\right)$ of our calculated results are for the most part somewhat larger than the experimental uncertainties shown in Fig. 1. However, in the temperature range where experimental data exist, our results (perhaps fortuitously) lie within the smaller uncertainties of the data. Therefore, Eq. (5) can also be viewed as a correlating equation that agrees with the experimental data, but which extrapolates these data in a physically correct manner and can therefore be used in preference to the Hyland-Wexler correlation in the entire range. Equation (6) provides the expanded uncertainty in our calculation of $B_{\text {aw }}(T)$ for the entire temperature range. However, at near-ambient temperatures where accurate data exist (see Fig. 1), $B_{\text {aw }}$ is known from experiment with a smaller uncertainty than that given by Eq. (6), so it might be appropriate for uncertainty evaluations at those temperatures to use uncertainties commensurate with those of the experimental data.

In computing our expanded uncertainties, we considered only contributions from the individual gas-water second virial coefficients. The only other possible contribution comes from the mole fractions of air components in Eq. (3). However, the composition of air is known quite accurately, and the effects of small changes in composition (including the effects of carbon dioxide and other trace components) are negligible compared to the uncertainties in the individual virial coefficients. This remains the case even if one accepts recent evidence $[13,14]$ that the mole fraction of argon in air is slightly larger than that in the current standard [11] (with a corresponding reduction in the nitrogen mole fraction). 
Further reduction of the uncertainty of our results would require reducing the uncertainty of $B(T)$ for the individual gas-water pairs by reducing the uncertainty in the potential-energy surfaces for those pairs. Because of its larger mole fraction, improvements in the $\mathrm{H}_{2} \mathrm{O}-\mathrm{N}_{2}$ surface would have the most impact. However, the $\mathrm{H}_{2} \mathrm{O}-\mathrm{O}_{2}$ pair also offers significant room for improvement, because that potential [10] is known with somewhat less relative accuracy than is the $\mathrm{H}_{2} \mathrm{O}-\mathrm{N}_{2}$ potential.

\section{CONCLUSIONS}

We have used results from computational quantum chemistry to derive the air-water second virial coefficient $B_{\text {aw }}$ from first principles. Our calculated results are in excellent agreement with the available experimental data, but cover a much wider temperature range. Equation (5) is recommended to replace the correlation of Hyland and Wexler [6] for calculating $B_{\mathrm{aw}}(T)$ and the enthalpy coefficient $\phi_{\mathrm{aw}}(T)$ in the temperature range from 150 to $2000 \mathrm{~K}$.

\section{ACKNOWLEDGMENTS}

The group of Richard Wheatley at the University of Nottingham developed the pair potentials of Refs. 8-10, which were essential to this project, and also provided additional calculations for the uncertainty analysis in Section 4. We thank Eric Lemmon of NIST for assistance in fitting several equations, and Michael Moldover of NIST for helpful discussion of uncertainties.

\section{REFERENCES}

1. X. Ji, X. Lu, and J. Yan, Appl. Thermal Eng. 23:2213 (2003).

2. H. F. Nelson and H. J. Sauer, Jr., J. Thermophys. Heat Transfer 18:135 (2004).

3. R. W. Hyland and A. Wexler, J. Res. Nat. Bur. Stand. (U.S.) 77A:133 (1973).

4. R. W. Hyland, J. Res. Nat. Bur. Stand. (U.S.) 79A:551 (1975).

5. R. G. Wylie and R. S. Fisher, J. Chem. Eng. Data 41:133 (1996).

6. R. W. Hyland and A. Wexler, ASHRAE Trans. 89:520 (1983).

7. J. O. Hirschfelder, C. F. Curtiss, and R. B. Bird, Molecular Theory of Gases and Liquids (John Wiley \& Sons, New York, 1954).

8. M. P. Hodges, R. J. Wheatley, and A. H. Harvey, J. Chem. Phys. 117:7169 (2002)

9. A. S. Tulegenov, R. J. Wheatley, M. P. Hodges, and A. H. Harvey, J. Chem. Phys. 126:094305 (2007).

10. R. J. Wheatley and A. H. Harvey, submitted to J. Chem. Phys. in press

11. P. Giacomo, Metrologia 18:33 (1982).

12. M. P. Hodges, R. J. Wheatley, G. K. Schenter, and A. H. Harvey, J. Chem. Phys. 120:710 (2004). 
13. S. Y. Park, J. Y. Kim, J. B. Lee, M. B. Esler, R. S. Davis, and R I. Wielgosz, Metrologia 41:387 (2004).

14. A. Picard, H. Fang, and M. Gläser, Metrologia 41:396 (2004). 